\title{
Diagnostic value of the interferon- $\gamma$ release assay for tuberculosis infection in patients with Behçet's disease
}

\author{
Xiuhua $\mathrm{Wu}^{1,2+}{ }^{+}$, Pang Chen ${ }^{3+}$, Wei Wei ${ }^{2}$, Mengyu Zhou' ${ }^{1}$, Chaoran $\mathrm{Li}^{1}$, Jinjing Liu' ${ }^{1}$, Lidan Zhao ${ }^{1}$, Lifan Zhang ${ }^{4}$, \\ Yan Zhao', Xiaofeng Zeng ${ }^{1}$, Xiaoqing Liu ${ }^{4^{*}}$ and Wenjie Zheng ${ }^{*^{*}}$ (D)
}

\begin{abstract}
Background: To investigate the diagnostic value of the interferon- $\gamma$ release assay (IGRA) for detecting tuberculosis (TB) infection in patients with Behçet's disease (BD).

Methods: We retrospective analyzed the data collected from 173 BD patients hospitalized between 2010 and 2015. Ninety-nine healthy volunteers were enrolled as a control group. IGRA was performed using T-SPOT.TB. The diagnosis of active TB (ATB) was based on clinical, radiological, microbiological, histopathological information and the response to anti-TB therapy. Latent TB (LTB) infection was defined as asymptomatic patients with positive T-SPOT.TB.

Results: TB infection was documented in 59 BD patients (34.1\%). The sensitivity, specificity, positive predictive value, negative predictive value, positive likelihood ratio and negative likelihood ratio of T-SPOT.TB for the diagnosis of ATB were $88.9 \%, 74.8 \%, 29.1 \%, 98.3 \%, 3.53$ and 0.15 , respectively. The receiver-operating-characteristic curve demonstrated that spot-forming cells (SFCS) of 70/10 $\mathrm{PBMC}$ was the optimal cutoff for diagnosing ATB, with an area under the curve of 0.891. Furthermore, the median SFCs in ATB group was significantly higher than those in LTB infection $\left(466 / 10^{6}\right.$

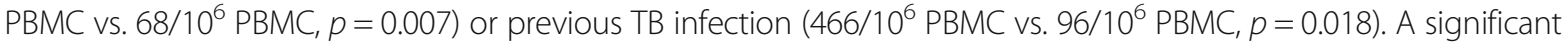
discrepancy between T-SPOT.TB and tuberculin skin test was noted (kappa coefficient $=0.391, p=0.002$ ).

Conclusions: T-SPOT.TB, an IGRA, may assist in the diagnosis of ATB in BD patients, and the higher SFCs suggest ATB in BD patients.
\end{abstract}

Keywords: Behçet's disease, Tuberculosis, Interferon- $\gamma$ release assay, T-SPOT.TB

\section{Background}

Behcet's disease (BD) is a multi-system inflammatory disorder, characterized by recurrent oral ulcers, genital ulcers, skin lesions, and ocular involvement of unknown etiology. Notably, the clinical manifestation of orogenital ulcers and skin lesions including erythema nodosum could be mimicked by tuberculosis (TB). Additionally,

\footnotetext{
*Correspondence: wenjzheng@gmail.com; liuxqpumch@126.com

${ }^{+}$Xiuhua Wu and Pang Chen contributed equally to this work.

'Department of Rheumatology and Clinical Immunology, Key Laboratory of Rheumatology and Clinical Immunology, Peking Union Medical College Hospital, Peking Union Medical College and Chinese Academy of Medical Sciences, Ministry of Education, Beijing, China

${ }^{4}$ Department of Infectious Diseases, Clinical Epidemiology Unit, International Epidemiology Network, Centre for Tuberculosis Research, Chinese Academy of Medical Sciences and Peking Union Medical College Hospital, Beijing, China

Full list of author information is available at the end of the article
}

active TB (ATB) is frequently observed in patients with $\mathrm{BD}$, and those symptoms were relieved after anti-TB therapy $[1,2] . \mathrm{BD}$ and $\mathrm{TB}$ are also closely related in pathogenesis. M.tuberculosis may act as a trigger of $\mathrm{BD}$ through molecular mimicking. On the other hand, defective cell-mediated immunity in BD patients may increase the susceptibility of TB [3], and the use of glucocorticoids, immunosuppressants or biologic agents may increase the risk of reactivation of TB [4]. Therefore, it is crucial and challenging to identify $\mathrm{TB}$ in $\mathrm{BD}$ patients.

Tuberculin skin test (TST), a routine screening test for latent TB (LTB) infection, has limited diagnostic value in $\mathrm{BD}$ patients due to cross-reactivity with the bacillus Calmette-Guérin (BCG) vaccine and nontuberculous mycobacteria as well as pathergy reaction [5]. In additions, the autoimmune disorder or immunosuppressive

(c) The Author(s). 2019 Open Access This article is distributed under the terms of the Creative Commons Attribution 4.0 International License (http://creativecommons.org/licenses/by/4.0/), which permits unrestricted use, distribution, and reproduction in any medium, provided you give appropriate credit to the original author(s) and the source, provide a link to the Creative Commons license, and indicate if changes were made. The Creative Commons Public Domain Dedication waiver (http://creativecommons.org/publicdomain/zero/1.0/) applies to the data made available in this article, unless otherwise stated. 
status could lead to false-negative test results [6]. Interferon- $\gamma$ release assays (IGRAs) are powerful assays for detection of TB [7], which detect the mycobacterialantigen-specific interferon- $\gamma$-releasing $\mathrm{T}$-cells in vitro. Given IGRAs do not cross-react with BCG vaccination or nontuberculous mycobacteria infection, IGRAs show higher specificity than TST [8]. IGRAs, including T-SPOT.TB, have been widely used to diagnose TB in autoimmune diseases, such as systemic lupus erythematosus (SLE) [8] and rheumatoid arthritis (RA) [9]. However, the diagnostic value of IGRAs for $\mathrm{TB}$ in $\mathrm{BD}$ patients remains unclear. To address this point, we retrospectively reviewed a cohort of $\mathrm{BD}$ patients tested with T-SPOT.TB and explored the diagnostic value of T-SPOT.TB for ATB in patients with BD.

\section{Methods}

\section{Patients}

Medical records of the hospitalized BD patients from Peking Union Medical College Hospital between January 2010 and March 2015 were retrospectively reviewed. All patients fulfilled the International Study Group BD criteria or the new International Criteria for $\mathrm{BD}[10,11]$. T-SPOT.TB was performed in BD patients when TB infection was suspected or corticosteroids and/or immunosuppressive drugs were planned to apply. We reviewed $268 \mathrm{BD}$ patients with available medical records and confirmed 173 patients performed with T-SPOT.TB test. Of the 173 patients, clinical, laboratory and radiology data were collected and analyzed, and BD disease activity was assessed using the BD Current Activity Form 2006 (BDCAF2006). A total of 99 age- and sex-matched healthy volunteers were served as controls during the same period. T-SPOT.TB was performed in all participants. The diagnosis of ATB was based on clinical manifestations, radiologic findings, microbiology and histopathology, and the response to anti-TB therapy $[12,13]$. ATB included culture-confirmed $\mathrm{TB}$ and the highly-probable TB. Culture-confirmed TB was defined as suggestive clinical and radiological findings and positive culture of M.tuberculosis. Highly-probable TB was defined as highly suggestive clinical and radiological features for $\mathrm{TB}$ in combination with the appropriate response to anti-TB treatment [12]. LTB infection was defined as asymptomatic patients with positive T.SPOT.TB [13].

\section{Interferon- $\gamma$ release assays (IGRAs)}

IGRAs were performed using T-SPOT.TB, a simplified enzyme-linked immunospot (ELISPOT) for detection of effector $\mathrm{T}$ cells that respond to stimulation by antigens specific for M.tuberculosis. Briefly, peripheral blood mononuclear cells were isolated from whole blood samples by density gradient separation, then stimulated with two TB specific antigens, early secreted antigenic target
6 (ESAT-6) and culture filtrate protein 10 (CFP-10), phytohaemagglutinin (PHA) (positive control) or AIM-V (GIBCOTMAIMV Medium liquid, Invitrogen, US) (negative control) in microplate wells $\left(2.5 \times 10^{5}\right.$ per well) precoated with interferon- $\gamma($ IFN- $\gamma$ ) capture antibodies. The number of spot-forming cells (SFCs) was calculated after 16 to $20 \mathrm{~h}$ of incubation. The result was considered positive if the number of SFCs was six or more after subtracting the spot count of negative control [14].

\section{Tuberculin skin test (TST)}

TST was performed with an intradermal injection using standardized $0.1 \mathrm{ml}(5 \mathrm{U})$-purified protein derivative into the ventral surface of the forearm according to the Mantoux method [15]. On the basis of National Institute for Health and Care Excellence (NICE) guidelines in 2016, the diameter of induration was measured in 48 to $72 \mathrm{~h}$ after administration $5 \mathrm{~mm}$ or more was defined as positive TST [16].

\section{Statistical analysis}

Numerical data were expressed as mean \pm standard deviation or median (interquartile range, IQR), and categorical data were expressed as frequencies/ percentages. The Mann-Whitney U test and Pearson chi-square test were used to compare differences between two groups. The concordance of TST and T-SPOT.TB results were assessed using каppa coefficient. (карра $>0.75$, excellent agreement; 0.40 to 0.75 , fair to good agreement; and < 0.40 , poor agreement). The diagnostic performance of T-SPOT.TB on ATB was evaluated by calculation of its sensitivity, specificity, positive predictive value (PPV), negative predictive value (NPV), positive likelihood ratio (PLR), and negative likelihood ratio (NLR). The area under the receiver operating characteristic curve of the T-SPOT.TB on peripheral blood mononuclear cell (PBMC) diagnostic cutoff for ATB was calculated. A two-sided with $p$ values $<0.05$ is considered statistically significant. Statistical analysis was performed by using SPSS (version 20.0, SPSS Inc., Chicago, IL, USA) and the GraphPad Prism (Version 5.01, GraphPad Software Inc., CA, USA).

\section{Results}

Characteristics of the study population

Among the $173 \mathrm{BD}$ patients performed with TB-SPOT.TB test, 114 (65.9\%) were men. The mean age was $37.07 \pm 14.74$ years, and median disease duration of BD was 84 months (range 1-608). The most common clinical manifestation was recurrent oral ulceration (98.3\%). Other common findings included fever (81.5\%), genital ulcers $(58.4 \%)$, skin lesions $(58.4 \%)$, gastrointestinal involvement (39.3\%), vascular involvement (28.3\%), positive pathergy test (22.5\%), ocular involvement (19\%), 
neurologic involvement (15\%), cardiac involvement (9.8\%) and hematological involvement (5.2\%). The median BDCAF2006 score for disease activity was 2 (range 0-5). Elevated erythrocyte sedimentation rate (ESR) and high-sensitivity C-reactive protein (hs-CRP) levels were detected in $98(56.6 \%)$ and 118 patients (68.2\%), respectively. The median ESR was 21 (range 1140) $\mathrm{mm}$ /first hour and the median hs-CRP was 11.54 (range $0.12-262.77) \mathrm{mg} / \mathrm{L}$. In the past 2 years, 113 patients $(65.3 \%)$ were treated with glucocorticoids, including 13 patients (7.5\%) received glucocorticoid pulse therapy. Ninety-seven patients (56.1\%) were under glucocorticoids treatment when T-SPOT.TB assay was performed, and the median daily dose of corticosteroids was 40 (range 5-100) mg. Ninety-four patients received immunosuppressants, including cyclophosphamide (30.1\%), leflunomide (5.2\%), cyclosporin A (4.6\%), azathioprine (4.6\%), methotrexate (4.6\%) and salazosulfapyridine $(2.9 \%)$. Sixteen patients $(9.2 \%)$ received two or more immunosuppressants. Biological agents were used in 12 patients (6.9\%), including infliximab injection in six patients, etanercept injection in five patients, etanercept followed by infliximab in one patient (Table 1).

\section{TB infection in BD patients}

TB infection was documented in 59 BD patients (34.1\%) in our study, including 18 (10.4\%) ATB (BD-ATB), 29 (16.8\%) LTB (BD-LTB) infection, and 12 (6.9\%) patients with evidence of previous TB. Of the $18 \mathrm{BD}-\mathrm{ATB}$ cases, culture-confirmed $\mathrm{TB}$ and highly probable $\mathrm{TB}$ were diagnosed in 4 and 14 patients, respectively. Of the four culture-confirmed TB patients, pulmonary TB, tuberculous lymphadenitis, tuberculous pleuritis, tuberculous meningitis were diagnosed in one patient, respectively. Among 14 highly-probable TB, highly-probable pulmonary TB was diagnosed in 7 cases based on fever, cough, expectoration or chest pain, and CT-confirmed pulmonary cavity, infiltrating or nodules as well as mediastinal lymphadenopathy. Highly-probable tuberculous pericarditis was diagnosed in 2 cases presenting with fever and bloody pericardial effusion. Highly-probable tuberculosis of the knee joint was diagnosed in one case presenting with fever, swollen and painful knee, radiography showed the effusion, synovial thickening, and bone erosions in the knee. The other four patients had a history of TB, and presented with persistent fever, effusion or lymphadenopathy which are uncommon manifestations in BD. All cases with highly-probable TB had a good response to anti-TB treatment, but not corticosteroids and immunosuppressive drugs. Twenty-one patients were followed-up for $72.31 \pm 16.45$ months, and no ATB was observed. The clinical characteristics of different groups of BD patients were presented in Table 1. Interestingly, BD-ATB patients showed more tendency in developing skin lesions $(72.2 \%$ vs $34.5 \%, p=0.012)$, especially erythema nodosa ( $50 \%$ vs $20.6 \%, p=0.036)$ compare to BD-LTB infection patients.

Table 1 Demographics and Clinical characteristics of 173 patients with Behcet's disease

\begin{tabular}{|c|c|c|c|c|c|}
\hline Characteristics & $\mathrm{BD}(n=173)$ & $\operatorname{ATB}(n=18)$ & $\operatorname{LTB}(n=29)$ & Previous TB $(n=12)$ & Non-TB $(n=114)$ \\
\hline Age (years, mean \pm SD) & $37.07 \pm 14.74$ & $36.78 \pm 10.61$ & $43.52 \pm 15.23$ & $42.75 \pm 14.06$ & $34.87 \pm 14.76$ \\
\hline Male (percentage) & $114(65.9 \%)$ & $11(61.1 \%)$ & $23(79.3 \%)$ & $5(41.7 \%)$ & 75 (65.8\%) \\
\hline BDCAF score (median, range) & $2(0-5)$ & $1.5(1-5)$ & $2(0-5)$ & $3(0-5)$ & $2(0-5)$ \\
\hline \multicolumn{6}{|l|}{ Clinical features of BD } \\
\hline Oral ulcer & $170(98.3 \%)$ & $18(100 \%)$ & $28(96.6 \%)$ & $12(100 \%)$ & $112(98.2 \%)$ \\
\hline Genital ulcer & $101(58.4 \%)$ & $15(83.3 \%)$ & $20(68.9 \%)$ & $9(75 \%)$ & $57(50 \%)$ \\
\hline Skin lesions & $101(58.4 \%)$ & $13(72.2 \%)^{a}$ & $10(34.5 \%)$ & $10(83.3 \%)$ & $68(59.6 \%)$ \\
\hline Erythema nodosa & $53(30.6 \%)$ & $9(50 \%)^{a}$ & $6(20.6 \%)$ & $5(41.6 \%)$ & $33(28.9 \%)$ \\
\hline Pathergy reaction & 39 (22.5\%) & 7 (38.9\%) & 7 (24.1\%) & $3(25 \%)$ & $22(19.3 \%)$ \\
\hline Gastrointestinal involvement & $68(39.3 \%)$ & $5(27.7 \%)$ & $12(41.4 \%)$ & $5(41.7 \%)$ & $46(40.3 \%)$ \\
\hline Vascular involvement & 49 (28.3\%) & $7(38.9 \%)$ & $9(31 \%)$ & $3(25 \%)$ & $30(26.3 \%)$ \\
\hline Ocular involvement & $33(19 \%)$ & $4(22.2 \%)$ & $3(10.3 \%)$ & $1(8.3 \%)$ & $25(21.9 \%)$ \\
\hline Neurologic involvement & $26(15 \%)$ & $1(5.5 \%)$ & $3(10.3 \%)$ & $3(25 \%)$ & 19 (16.7\%) \\
\hline \multicolumn{6}{|l|}{ Treatment } \\
\hline Glucocorticoid & $113(65.3 \%)$ & $12(66.7 \%)$ & $22(75.9 \%)$ & $6(50 \%)$ & 73 (64\%) \\
\hline Immunosuppressive agents & $94(54.3 \%)$ & $7(38.9 \%)$ & $16(55.2 \%)$ & $8(66.7 \%)$ & $63(55.3 \%)$ \\
\hline \multicolumn{6}{|l|}{ Biological agents } \\
\hline Infliximab/Etanercept & $12(6.9 \%)$ & $2(11.1 \%)$ & $2(6.9 \%)$ & $1(8.3 \%)$ & 7 (6.1\%) \\
\hline
\end{tabular}

ATB compare with LTB; ${ }^{a} p<0.05$ 
The diagnostic value of T-SPOT.TB for ATB in BD patients Positive T-SPOT.TB tests were detected in 16 patients with ATB (88.9\%), and the median number of spot-forming cells (SFCs) was 466/10 ${ }^{6}$ PBMC (IQR: 108-925). Among patients with evidence of previous $\mathrm{TB}$, ten patients $(83.3 \%)$ had positive T-SPOT.TB with the median number of SFCs being $96 / 10^{6}$ PBMC (IQR: 31.75-187). The median number of SFCs in BD-LTB infection patients was $68 / 10^{6}$ PBMC (IQR: 38-208). The median number of SFCs in the BD-ATB group was higher than those in the BD-LTB infection group ( $p=$ $0.007)$ or those in previous TB patients $(p=0.018)$. The median numbers of SFCs in the BD-ATB group, BD-LTB infection group and previous TB patients were higher than those in the non-TB infection group $(p=0.000)$ (Fig. 1).

The sensitivity, specificity, PPV and NPV of the T-SPOT.TB test for the diagnosis of ATB were $88.9 \%$ (95\%CI 63.9-98.1\%), 74.8\% (95\%CI 67.1-81.3\%), 29.1\% (95\%CI 18-43.1\%), and $98.3 \%$ (95\%CI 93.4-99.7\%), respectively. PLR was $3.53(95 \%$ CI $2.57-4.85)$ and NLR was 0.15 (95\%CI 0.04-0.55).

The receiver-operating-characteristic curve demonstrated that an SFCs of $70 / 10^{6}$ PBMC was the optimal cutoff for diagnosing BD-ATB, with an area under the curve of 0.891 (95\%CI 0.796-0.987) (Fig. 2). Accordingly, the sensitivity, specificity, PPV, NPV, PLR and NLR was $88.9 \%$ (95\%CI 63.9-98.1\%), 87.1\% (95\%CI 80.5-91.8\%), 44.4\% (95\%CI 28.3-61.7), 98.5\% (95\%CI 94.3-99.7\%), 6.89 (95\%CI 4.43-10.7), and 0.13 (95\%CI 0.03-0.47), respectively.

LTB infection was documented in 27(27.3\%) healthy controls, and the median number of SFCs was $192 / 10^{6}$

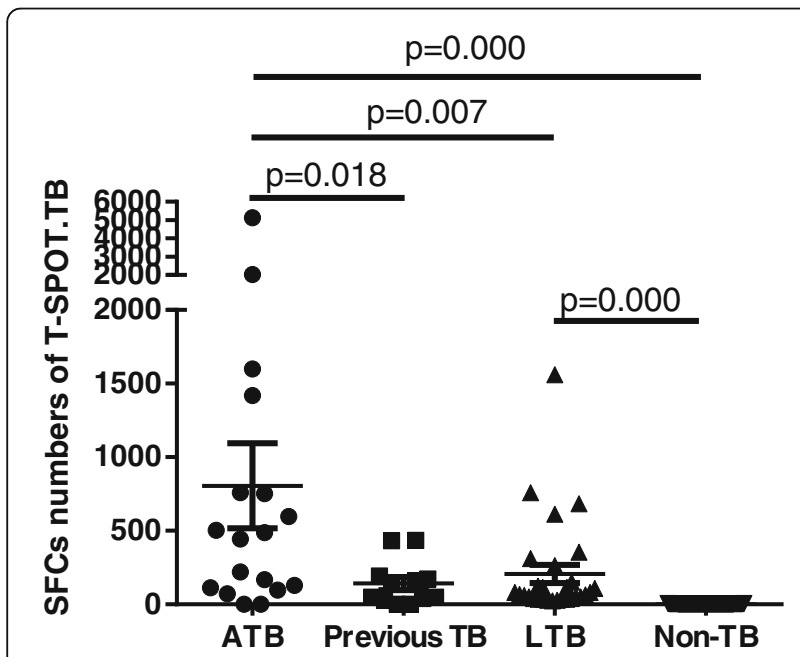

Fig. 1 Comparison the number of SFCs between different groups of BD patients. The median SFCs of ATB $(n=18)$, previous TB $(n=12)$, LTB $(n=29), \operatorname{Non}-\mathrm{TB}(n=114)$ was 466 (IQR: 108-925), 96 (IQR: 31.75-187), 68 (IQR: 38-208), and 2 (IQR: 0-3), respectively
PBMC (IQR:80-308). There was no significant difference in the proportion of LTB infection (20.3\% vs $27.3 \%$, $p=0.205)$ and the number of SFCs detected by T-SPOT.TB $\left(68 / 10^{6}\right.$ PBMC vs $192 / 10^{6}$ PBMC, $\left.p=0.079\right)$ between $\mathrm{BD}$ patients and healthy controls.

\section{The diagnostic values of T-SPOT.TB and TST for ATB}

Both T-SPOT.TB and TST results were simultaneously tested in 63 patients. Among these patients, positive results of TST and T-SPOT.TB were presented in $44.4 \%$ $(28 / 63)$ and $46 \%(29 / 63)$ patients, respectively. $30.2 \%$ $(19 / 63)$ of the 63 patients had both positive TST and T-SPOT.TB, and $60.3 \%(38 / 63)$ had positive results on either TST or T-SPOT.TB (Table 2). A significant discrepancy between T-SPOT.TB and TST was noted (kappa coefficient of $0.391,95 \%$ CI $0.146-0.617, p=$ 0.002).

The sensitivity of TST for detecting ATB was $78.6 \%$ (95\%CI 48.8-94.3\%), which was lower than that of the T-SPOT.TB test $(92.9 \%)$, but there was no significant difference between the two groups. The specificity of TST was similar with T-SPOT.TB test (Table 3).

We also evaluated the diagnostic values of the combination of T-SPOT.TB and TST for ATB diagnosis in these 63 patients (Table 3) with parallel or serial manner, which was defined as either test was positive or both tests were positive, respectively. Serial test improved the specificity of diagnosis of ATB to $83.7 \%$ when combined T-SPOT.TB with TST, while no statistical significance was shown $(67.3 \%$ vs $83.7 \%, p=0.06)$. However, there was no difference in the sensitivity between parallel testing and T-SPOT.TB assay alone.

\section{Discussion}

$\mathrm{TB}$ is an infectious disease primarily involving lungs and is endemic in developing countries. China has the world's second largest TB prevalence, comprising around 9\% of the world total according to the 2018 World Health Organization (WHO) TB report [17]. Patients with rheumatic diseases at high risk of TB infection, due to the immune dysregulation and the adverse effects of immunosuppressive agents [18-20]. M.tuberculosis culture, the gold standard of TB diagnosis usually takes 2-4 weeks. Therefore, a rapid diagnostic method of TB remains challenging. TST is a valuable screening test for TB infection [21], but the diagnostic value of TST in BD patients is compromised by the widely usage of the BCG vaccine in Chinese population and the immune dysfunction in BD patients [22].

In recent years, IGRAs emerges as accurate diagnostic assays for screening of TB infection. IGRAs specifically detect the presence of specific effector $\mathrm{T}$ cells which response to M.tuberculosis-specific antigens [23]. Dai Y. et al. systematically evaluated the diagnostic accuracy of 


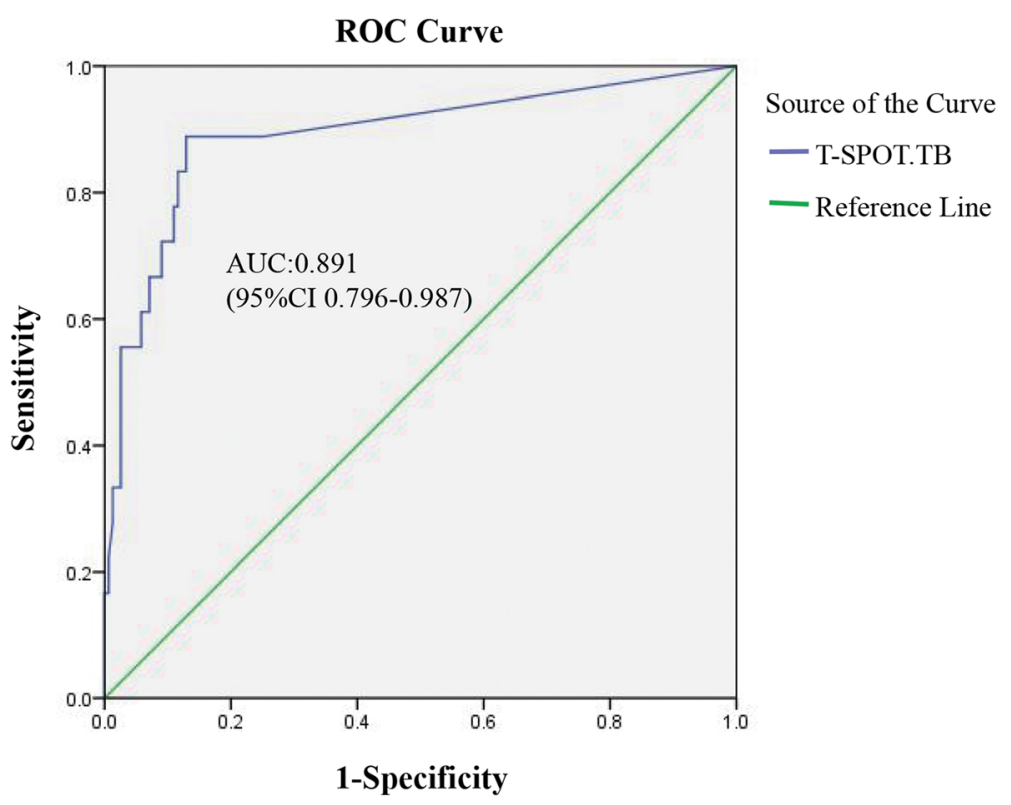

Fig. 2 Receiver-operating-characteristic curve of T -SPOT.TB test in patients with Behcet's disease

T-SPOT.TB for detecting ATB in China, and found that the pooled sensitivity, specificity, PLR and NLR of T-SPOT.TB for the diagnosis of ATB were $88 \%$ (95\%CI:86-91\%), 89\% (95\%CI: 86-92\%), 8.86 (95\%CI: 5.42-14.46), and 0.13 (95\% CI: $0.10-0.17$ ), respectively [24]. And The ROC area under the curve (AUC) was 0.9548 (95\% CI: 0.9323-0.9773) [24]. Consistently, Jiang $\mathrm{B}$, et al. find that the sensitivity and specificity of T-SPOT.TB assays were 92.86 and $93.64 \%$, in patients with rheumatic disease, which were higher than TST [25].

In this study, we evaluated T-SPOT.TB and TST for the diagnosis of ATB in BD patients. To our knowledge, this is the first study that reports the diagnostic value of T-SPOT.TB for TB in BD patients. We reported the high sensitivity (88.9\%) and specificity (74.8\%) of T-SPOT.TB for the diagnosis of ATB in BD patients. Compared to Dai Y's study [24], our study showed similar results of sensitivity and NLR of the T-SPOT.TB test for the diagnosis of ATB while the lower specificity and PLR. The different study populations may explain this difference. Using SFCs $>70 / 10^{6} \mathrm{PBMC}$ as the cutoff, the specificity and PLR are improved for diagnosing ATB in BD

Table 2 Discrepancy between T-SPOT.TB and TST (in 63 BD patients)

\begin{tabular}{lllll}
\hline & & \multicolumn{2}{l}{ T-SPOT.TB } & \\
\cline { 3 - 4 } & & Positive & Negative & Total \\
\hline TST & Positive & 19 & 9 & 28 \\
& Negative & 10 & 25 & 35 \\
& Total & 29 & 34 & 63 \\
\hline
\end{tabular}

TST tuberculin skin test patients. Although, neither T-SPOT.TB nor TST can clearly distinguish between LTB infection, ATB or history of TB infection [23, 26], we found the median number of SFCs in BD-ATB group was higher than those in BD-LTB infection group and previous TB group, which suggested that higher SFCs in T-SPOT.TB test might indicate ATB. The numbers of IFN- $\gamma$ producing $\mathrm{T}$ cells decreased during anti-tuberculous treatment, but IGRAs results remained positive at the end of treatment in most patients, which suggest a limited role of IGRAs in monitoring treatment efficacy [27]. Meanwhile, particular attention should be paid to ATB with negative IGRAs results, since those patients had poor outcomes, potentially due to delayed treatment [28].

It has been reported that the pooled sensitivity of T-SPOT.TB was higher than that of TST for the diagnosis of ATB in culture-confirmed or non-confirmed TB [29]. A discrepancy between T-SPOT.TB and TST test was noted in our study. The serial testing could increase the specificity of T-SPOT.TB for ATB diagnosis in BD patients when combined with TST, but the result didn't reach statistical differences due to a limited number of cases. Further studies were required to explore the diagnostic efficiency between T-SPOT.TB and TST.

LTB infection is defined as the sustained immune response to Mycobacterium tuberculosis antigens with no evidence of clinically ATB. Given the risk of LTB infection reactivation increases in patients receiving immunosuppressive therapy or with immune dysfunction [30], it is important to identify LTB infection in BD patients. However, currently, there is no gold standard test for diagnosing LTB infection available. Several studies 
Table 3 Comparison the diagnostic values between T-SPOT.TB and TST and their combinations

\begin{tabular}{lllllll}
\hline & $\begin{array}{l}\text { Sensitivity } \\
(\%, 95 \mathrm{Cl})\end{array}$ & $\begin{array}{l}\text { Specificity } \\
(\%, 95 \mathrm{Cl})\end{array}$ & $\begin{array}{l}\text { Positive predictive } \\
\text { value }(\%, 95 \mathrm{Cl})\end{array}$ & $\begin{array}{l}\text { Negative predictive } \\
\text { value }(\%, 95 \mathrm{Cl})\end{array}$ & $\begin{array}{l}\text { Positive likelihood } \\
\text { ratio }(95 \% \mathrm{Cl})\end{array}$ & $\begin{array}{l}\text { Negative likelihood } \\
\text { ratio (95\%Cl) }\end{array}$ \\
\hline T-SPOT.TB & $92.9(64.2-99.6)$ & $67.3(52.3-79.6)$ & $44.8(27-64)$ & $97(82.5-99.8)$ & $2.84(1.85-4.36)$ & $0.11(0.0160 .71)$ \\
TST & $78.6(48.8-94.3)$ & $65.3(50.2-77.9)$ & $39.2(22.1-59.3)$ & $91.4(75.8-97.8)$ & $2.26(1.41-3.63)$ & $0.32(0.12-0.91)$ \\
TSPOT.TB and TST (parallel) & $92.9(64.2-99.6)$ & $48.9(34.6-63.5)$ & $34.2(20.1-51.4)$ & $96(77.7-99.8)$ & $1.82(1.33-2.48)$ & $0.15(0.02-1.01)$ \\
TSPOT.TB and TST (serial) & $78.5(48.8-94.3)$ & $83.7(69.8-92.2)$ & $57.8(34-78.9)$ & $93.2(80.3-98.2)$ & $4.81(2.41-9.6)$ & $0.26(0.09-0.70)$ \\
\hline
\end{tabular}

TST tuberculin skin test

[31-33] suggested that IGRAs, including QuantiFERONTB Gold In-Tube (QFT-GIT) and T-SPOT.TB, and TST, are all acceptable for screening LTB infection. In immune-mediated inflammatory diseases, the sensitivity and specificity of IRGAs in the diagnosis of LTB infection is higher than those in TST [34-36]. Also, T-SPOT.TB has been reported with higher sensitivity in detecting LTB infection than QFT-GIT and TST [32, 37], and Vassilopoulos et al. reported that the positive rates of QFT-GIT and T-SPOT.TB were 21 and 25\%, respectively [38] . In our study, patients who had positive $\mathrm{T}$-SPOT.TB but did not have ATB or history of TB infection were considered as LTB infection. The percentage of LTB infection in BD patients in our study was similar to that in other studies $[32,38]$. A notable concern of IGRA in BD patients is the false positive or false negative rates of the IGRA test. Firstly, immunosuppressive agents or glucocorticoid might suppress $\mathrm{T}$ cell response to $\mathrm{TB}$ antigens and cause false negative IGRA test in $\mathrm{BD}$ patients. Nevertheless, a meta-analysis of IGRA in rheumatic patients showed that neither steroid nor disease-modifying anti-rheumatic drugs (DMARDs) significantly affect positive IGRA results [39]. Secondly, in BD patients with high disease activity, the systemic inflammation in $\mathrm{BD}$ potentially promotes $\mathrm{T}$ cell response via high-level proinflammatory cytokines. However, $\mathrm{T}$ cell response detected in IGRA is derived from the $\mathrm{T}$ cells with specific $\mathrm{TCR}$ recognizing tuberculosis-specific peptide, and systemic inflammation would not promote antigen-specific $\mathrm{T}$ cell response in the absence of specific peptide. Consistently, the SFCs of the negative control was less than 10 spots in our patients, which was comparable to those in healthy controls. Therefore, positive IGRA is strongly suggestive for latent $\mathrm{TB}$ in BD patients.

Our study has several limitations. First, our study is a retrospective analysis of a case series, which may have incomplete data of TST. Second, since all BD patients were enrolled from a single-center with relatively small sample sizes, and our center is a national referred center for complicated rheumatic diseases, potential selection bias of $\mathrm{BD}$ patients is possible. Third, it is challenging to identify active $\mathrm{TB}$ in $\mathrm{BD}$ patients based on clinical features since systemic inflammation is a shared feature of both BD and TB. The majority of our ATB patients were diagnosed according to clinical criteria rather than M.tuberculosis bacteriologically confirmed, which might introduce a high risk of bias. Fourth, given no gold standard of LTB infection is available, the precision of the diagnosis of LTB infection by T-SPOT.TB could not be assessed in this study. Nevertheless, this is the first study on the diagnostic value of T-SPOT.TB for TB in BD patients. A large-scale, multi-center study enrolled bacteriologically confirmed TB patients are warranted to confirm our findings.

\section{Conclusions}

T-SPOT.TB may assist in the diagnosis of ATB in BD patients, and the higher number of SFCs $\left(>70 / 10^{6}\right.$ PBMC) strongly suggested ATB. Further study is needed to investigate the diagnostic value of T-SPOT.TB for LTB infection in BD patients.

\section{Abbreviations \\ ATB: Active tuberculosis; BCG: Bacillus Calmette-Guérin; BD: Behcet's disease; CFP-10: Culture filtrate protein 10; CRP: C-reactive protein; ELISPOT: Enzyme- linked immunospot; ESAT-6: Early secreted antigenic target 6; \\ ESR: Erythrocyte sedimentation rate; IFN- $\gamma$ : Interferon- $\gamma$; IGRAs: Interferon- $\gamma$ release assays; IQR: Interquartile range; LTB: Latent tuberculosis; \\ NICE: National Institute for Health and Care Excellence; NLR: Negative likelihood ratio; NPV: Negative predictive value; PBMC: Peripheral blood mononuclear cell; PHA: Phytohaemagglutinin; PLR: Positive likelihood ratio; PPV: Positive predictive value; QFT-GIT: QuantiFERON-TB Gold In-Tube; RA: Rheumatoid arthritis; SFCs: Spot-forming cells; SLE: Systemic lupus erythematosus; TB: Tuberculosis; TST: Tuberculin skin test}

\section{Acknowledgements}

We thank the health professional staff from Department of Rheumatology \& Clinical Immunology, Peking Union Medical College Hospital and the patients for their participation in this study.

\section{Funding}

This study was supported by grants from the National Natural Science Foundation of China [grant number 81571598 and 81871299]; National Key Research and Development Program: "Precise Medical Research" [grant number 2016YFC0906201]; CAMS Initiative for Innovative Medicine [grant number 2016-I2M-1-013]; the National Major Science and Technology Research Projects for the Control and Prevention of Major Infectious Diseases in China [grant number 2014ZX10003003] and National Health and Family Planning Commission of the People's Republic of China [grant number 201402001]. The funders had no role in study design, data collection, data analysis, and data interpretation or writing of the manuscript.

\section{Availability of data and materials}

The datasets generated and/or analysed during the current study are not publicly available due to anonymity policy issues but are available from the corresponding author on reasonable request. 


\section{Authors' contributions}

All authors made substantial contributions to the conception and design of this study. XHW and PC acquired the data. XHW and LDZ performed the data analysis and interpretation. XHW wrote the manuscript. WJZ provided critical revisions to the manuscript. MYZ, JJL, WW, LFZ, CRL, YZ, XFZ, XQL also critically reviewed the manuscript and provided valuable input. All authors read and approved the final manuscript.

\section{Ethics approval and consent to participate}

The study was approved by the institutional review board of Peking Union Medical College Hospital (approval number: S-443) and all methods were performed in accordance with the relevant guidelines and regulations. All patients provided written informed consent in accordance with the Declaration of Helsinki.

\section{Consent for publication}

Not applicable.

\section{Competing interests}

The authors declare that they have no competing interests.

\section{Publisher's Note}

Springer Nature remains neutral with regard to jurisdictional claims in published maps and institutional affiliations.

\section{Author details \\ 'Department of Rheumatology and Clinical Immunology, Key Laboratory of Rheumatology and Clinical Immunology, Peking Union Medical College Hospital, Peking Union Medical College and Chinese Academy of Medical Sciences, Ministry of Education, Beijing, China. ${ }^{2}$ Department of Rheumatology, Tianjin Medical University General Hospital, Tianjin, China. ${ }^{3}$ Department of Rheumatology, Affiliated Mindong Hospital of Fujian Medical University, Fuan, Fujian, China. ${ }^{4}$ Department of Infectious Diseases, Clinical Epidemiology Unit, International Epidemiology Network, Centre for Tuberculosis Research, Chinese Academy of Medical Sciences and Peking Union Medical College Hospital, Beijing, China.}

Received: 1 August 2018 Accepted: 4 April 2019

Published online: 15 April 2019

\section{References}

1. Hamill M, Remedios D, Kapembwa M. Orogenital ulceration with overlapping tuberculosis: epiphenomenon or expanding spectrum of Behcet disease? J Low Genit Tract Dis. 2006;10:219-22.

2. Shinoda K, Hayashi R, Taki H, Hounoki H, Makino T, Nomoto K, et al. PseudoBehcet's disease associated with tuberculosis: a case report and review of the literature. Rheumatol Int. 2014;34:1471-4.

3. Efthimiou J, Hay PE, Spiro SG, Lane DJ. Pulmonary tuberculosis in Behçet's syndrome. Br J Dis Chest. 1988:82:300-4.

4. Ormerod P, Milburn HJ, Gillespie S, Ledingham J, Rampton D. BTS recommendations for assessing risk, and for managing M.tuberculosis infection and disease, in patients due to start anti-TNF treatment. Cytokine. 2005;60:800.

5. Farhat M, Greenaway C, Pai M, Menzies D. False-positive tuberculin skin tests: what is the absolute effect of BCG and non-tuberculous mycobacteria? Int J Tuberc Lung Dis. 2006;10:1192-204.

6. León DPD, Acevedovásquez E, Sáncheztorres A, Cucho M, Alfaro J, Perich R, et al. Attenuated response to purified protein derivative in patients with rheumatoid arthritis: study in a population with a high prevalence of tuberculosis. Ann Rheum Dis. 2005;64:1360.

7. Pai M, Denkinger CM, Kik SV, Rangaka MX, Zwerling A, Oxlade O, et al. Gamma interferon release assays for detection of mycobacterium tuberculosis infection. Clin Microbiol Rev. 2014;27:3-20.

8. Takeda N, Nojima T, Terao C, Yukawa N, Kawabata D, Ohmura K, et al. Interferon-gamma release assay for diagnosing mycobacterium tuberculosis infections in patients with systemic lupus erythematosus. Lupus. 2011;20: 792-800.

9. Kowada A. Cost effectiveness of interferon-gamma release assay for tuberculosis screening of rheumatoid arthritis patients prior to initiation of tumor necrosis factor-alpha antagonist therapy. Mol Diagn Ther. 2010;14: 367-73.
10. International Study Group for Behçet's Disease. Criteria for diagnosis of Behcet's disease. Lancet. 1990;335:1078-80.

11. International Team for the Revision of the International Criteria for Behçet's Disease. The International Criteria for Behçet's Disease (ICBD): a collaborative study of 27 countries on the sensitivity and specificity of the new criteria. J Eur Acad Dermatol Venereol. 2014;28:338.

12. Dosanjh DP, Hinks TS, Innes JA, Deeks JJ, Pasvol G, Hackforth S, et al. Improved diagnostic evaluation of suspected tuberculosis. Ann Intern Med. 2008;148:325-36.

13. Identificatio H. Diagnostic standards and classification of tuberculosis in adults and children. Am J Respir Crit Care Med. 2000;161:1376-95.

14. Meier T, Eulenbruch HP, Wrighton-Smith P, Enders G, Regnath T. Sensitivity of a new commercial enzyme-linked immunospot assay (T SPOT-TB) for diagnosis of tuberculosis in clinical practice. Eur J Clin Microbiol Infect Dis. 2005:24:529-36.

15. Deck F, Guld J. The WHO tuberculin test. Bull Int Union Tuberc. 1964;34:53-70.

16. Hoppe LE, Kettle R, Eisenhut M, Abubakar I. Guideline development group. Tuberculosis--diagnosis, management, prevention,and control: summary of updated NICE guidance. BMJ. 2016;352:h6747.

17. Organization WH. Global Tuberculosis Report 2018 Global Tuberculosis Report; 2018. p. 9

18. Carmona L, Hernandez-Garcia C, Vadillo C, Pato E, Balsa A, Gonzalez-Alvaro I, et al. Increased risk of tuberculosis in patients with rheumatoid arthritis. J Rheumatol. 2003;30:1436-9.

19. Bouza E, Moya JG, Munoz P. Infections in systemic lupus erythematosus and rheumatoid arthritis. Infect Dis Clin N Am. 2001;15:335-61.

20. Winthrop KL, Siegel JN, Jereb J, Taylor Z, lademarco MF. Tuberculosis associated with therapy against tumor necrosis factor alpha. Arthritis Rheum. 2005:52:2968-74.

21. Jr SD. The tuberculin skin test. Am Rev Respir Dis. 1982;125:108-18.

22. Wang L, Turner MO, Elwood RK, Schulzer M, Fitzgerald JM. A meta-analysis of the effect of Bacille Calmette Guérin vaccination on tuberculin skin test measurements. Thorax. 2002;57:804.

23. Ferrara G, Losi M, D'Amico R, Roversi P, Piro R, Meacci M, et al. Use in routine clinical practice of two commercial blood tests for diagnosis of infection with mycobacterium tuberculosis: a prospective study. Lancet. 2006;367:1328-34.

24. Dai Y, Feng $Y, X u R, X u$ W, Lu W, Wang J. Evaluation of interferon-gamma release assays for the diagnosis of tuberculosis: an updated meta-analysis. Eur J Clin Microbiol Infect Dis. 2012;31:3127-37.

25. Jiang B, Ding H, Zhou L, Chen X, Chen S, Bao C. Evaluation of interferongamma release assay (T-SPOT.TB $\left(\mathrm{T}^{\mathrm{M}}\right)$ ) for diagnosis of tuberculosis infection in rheumatic disease patients. Int J Rheum Dis. 2016;19:38-42.

26. Lange C, Pai M, Drobniewski F, Migliori GB. Interferon-gamma release assays for the diagnosis of active tuberculosis: sensible or silly? Eur Respir J. 2009;33:1250-3.

27. Vanessa $\mathrm{C}, \mathrm{Yu} \mathrm{H}$, Christel Z, Tom C, Nigel C. Interferon gamma release assays for monitoring the response to treatment for tuberculosis: a systematic review. Tuberculosis. 2015;95:639-50.

28. Nguyen DT, Teeter LD, Graves J, Graviss EA. Characteristics associated with negative interferon- $\gamma$ release assay results in culture-confirmed tuberculosis patients, Texas, USA, 2013-2015. Emerg Infect Dis. 2018;24:534.

29. Sester M, Sotgiu G, Lange C, Giehl C, Girardi E, Migliori GB, et al. Interferongamma release assays for the diagnosis of active tuberculosis: a systematic review and meta-analysis. Eur Respir J. 2011;37:100-11.

30. Small PM, Fujiwara PI. Management of tuberculosis in the United States. N Engl J Med. 2001;345:189-200.

31. Scrivo R, Sauzullo I, Mengoni F, laiani G, Vestri AR, Priori R, et al. Serial interferon-gamma release assays for screening and monitoring of tuberculosis infection during treatment with biologic agents. Clin Rheumatol. 2012;31:1567-75.

32. Minguez S, Latorre I, Mateo L, Lacoma A, Diaz J, Olive A, et al. Interferongamma release assays in the detection of latent tuberculosis infection in patients with inflammatory arthritis scheduled for anti-tumour necrosis factor treatment. Clin Rheumatol. 2012;31:785-94.

33. Trajman A, Steffen RE, Menzies D. Interferon-gamma release assays versus tuberculin skin testing for the diagnosis of latent tuberculosis infection: an overview of the evidence. Pulm Med. 2013;2013:601737.

34. Diel R, Goletti D, Ferrara G, Bothamley G, Cirillo D, Kampmann B, et al. Interferon-gamma release assays for the diagnosis of latent mycobacterium tuberculosis infection: a systematic review and meta-analysis. Eur Respir J. 2011;37:88-99. 
35. Mack U, Migliori GB, Sester M, Rieder HL, Ehlers S, Goletti D, et al. LTBI: latent tuberculosis infection or lasting immune responses to $\mathrm{M}$. tuberculosis? A TBNET consensus statement. Eur Respir J. 2009;33:956-73.

36. Smith R, Cattamanchi A, Steingart KR, Denkinger C, Dheda K, Winthrop KL, et al. Interferon-gamma release assays for diagnosis of latent tuberculosis infection: evidence in immune-mediated inflammatory disorders. Curr Opin Rheumatol. 2011;23:377-84.

37. Yu JJ, Woo HI, Jeon K, Koh WJ, Dong KJ, Cha HS, et al. The significance of sensitive interferon gamma release assays for diagnosis of latent tuberculosis infection in patients receiving tumor necrosis factor-a antagonist therapy. PLoS One. 2015;10:e0141033.

38. Vassilopoulos D, Tsikrika S, Hatzara C, Podia V, Kandili A, Stamoulis N, et al. Comparison of two gamma interferon release assays and tuberculin skin testing for tuberculosis screening in a cohort of patients with rheumatic diseases starting anti-tumor necrosis factor therapy. Clin Vaccine Immunol. 2011;18:2102-8

39. Ruan Q, Zhang S, Ai J, Shao L, Zhang W. Screening of latent tuberculosis infection by interferon- $\gamma$ release assays in rheumatic patients: a systemic review and meta-analysis. Clin Rheumatol. 2016;35:417-25.

Ready to submit your research? Choose BMC and benefit from:

- fast, convenient online submission

- thorough peer review by experienced researchers in your field

- rapid publication on acceptance

- support for research data, including large and complex data types

- gold Open Access which fosters wider collaboration and increased citations

- maximum visibility for your research: over $100 \mathrm{M}$ website views per year

At $\mathrm{BMC}$, research is always in progress.

Learn more biomedcentral.com/submissions 